\title{
Hybrid multilevel plane wave based near-field far-field transformation utilising combined near- and far-field translations
}

\author{
C. H. Schmidt ${ }^{1}$ and T. F. Eibert ${ }^{2}$ \\ ${ }^{1}$ Institute of Radio Frequency Technology, Universität Stuttgart, Germany \\ ${ }^{2}$ Lehrstuhl für Hochfrequenztechnik, Technische Universität München, Germany
}

\begin{abstract}
The radiation of large antennas and those operating at low frequencies can be determined efficiently by near-field measurement techniques and a subsequent nearfield far-field transformation. Various approaches and algorithms have been researched but for electrically large antennas and irregular measurement contours advanced algorithms with low computation complexity are required. In this paper an algorithm employing plane waves as equivalent sources and utilising efficient diagonal translation operators is presented. The efficiency is further enhanced using simple farfield translations in combination with the expensive nearfield translations. In this way a low complexity near-field transformation is achieved, which works for arbitrary sample point distributions and incorporates a full probe correction without increasing the complexity.
\end{abstract}

\section{Introduction}

Far-field antenna radiation pattern measurements of electrically large antennas or antennas at low operating frequencies are often complicated due to the large far-field distance. Near-field measurements can be performed in indoor test ranges of limited size and efficient near-field far-field transformation algorithms are known, which compute the desired radiation characteristic of the antenna under test (AUT) with high accuracy. Thereby the radiation behaviour of the AUT is modelled by equivalent sources. Various types of equivalent sources are feasible resulting in different near-field transformation algorithms all having their own benefits and drawbacks. Popular approaches are equivalent current methods (Sarkar and Taaghol, 1999), which are well suited for irregular and non-closed measurement grids as well as near-field samples close to the AUT. These methods assume an equivalent current distribution either on a fictitious surface or the

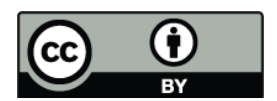

Correspondence to: $\mathrm{C}$. H. Schmidt (schmidt@ihf.uni-stuttgart.de) radiating structure itself and relate the currents to the nearfield values using an electric field integral equation. Another approach, implemented in various commercial tools, is based on eigenmode expansions (Hansen, 1988; Kerns, 1981) of the radiated AUT fields. The AUT fields are expanded in the eigenmodes of the employed measurement contour, namely plane waves for Cartesian grids, and cylindrical and spherical waves for cylindrical and spherical measurement contours. The orthogonality relations of the eigenmodes are used to relate the wave amplitudes to the measured near-field values. A detailed description of the different techniques is beyond the scope of this paper and an overview can be found in Yaghjian (1986).

The algorithm presented in this paper works with plane waves as equivalent sources, which are assumed for all directions on the Ewald sphere (Schmidt et al., 2008). One of the main advantages are the diagonal translation operators known from fast multipole methods (FMM) (Coifman et al., 1993; Chew et al., 2001), which are used to translate the plane waves from the AUT to the field probe position in an efficient way, where all waves are superimposed. Using a multilevel grouping scheme of the measurement points (Schmidt and Eibert, 2008), translations are carried out to the box centres on the highest level only and the plane wave contributions are processed towards the measurement points using a disaggregation and anterpolation scheme. Thereby a complexity of $\mathcal{O}(N \log N), N$ being the number of measurement points can be achieved. The unknown wave amplitudes are obtained in an inverse process. In this paper a hybrid approach is presented, which combines the efficient far-field translations with the standard, more expensive nearfield translations. Far-field translations can be utilised for measurement points fulfilling the far-field condition. The farfield criterion can be relaxed by subdividing the AUT in several source boxes each with a decreased far-field distance. This approach is not based on the evaluation of orthogonal basis functions and is thus able to deal with arbitrary and also irregular sample point distributions.

Published by Copernicus Publications on behalf of the URSI Landesausschuss in der Bundesrepublik Deutschland e.V. 


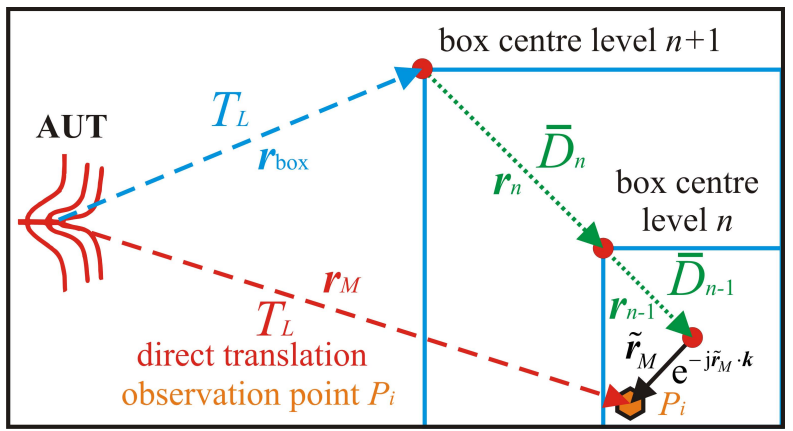

Fig. 1. Multilevel approach with disaggregation and anterpolation.

For practical measurements electric near-field values can be measured indirectly via the output voltage of a field probe only. The finite geometric extent and the receiving characteristic of the probe have a more or less strong impact on the measurement results, depending on the order of the probe. Therefore it is necessary to consider and compensate this influence in the near-field transformation to obtain accurate transformation results. This is called probe correction. For the well-known spherical multipole techniques different probe correction algorithms are available depending on the order of the probe and also the desired accuracy. A full correction of arbitrary probes becomes time consuming since either long transformation or measurement times are required. In the presented approach a full probe correction, similar to the classical plane wave approaches, is already included without increasing the complexity of the algorithm.

In Sect. 2 the multilevel near-field transformation algorithm based on plane wave expansion and diagonal translation operators is described. Section 3 introduces the hybrid approach utilising combined near- and far-field translations. Section 4 shows a performance evaluation of the presented method together with some results and Sect. 5 concludes the paper.

\section{Multilevel near-field far-field transformation}

The near-field transformation algorithm presented in this paper utilises plane waves $\tilde{\boldsymbol{J}}(\hat{k})$ on the entire Ewald sphere as equivalent sources. The output voltage (Schmidt et al., 2008)

$$
\begin{aligned}
\boldsymbol{U}\left(\boldsymbol{r}_{M}\right)= & -\mathrm{j} \frac{\omega \mu}{4 \pi} \oiiint T_{L}\left(\hat{k}, \hat{r}_{M}\right) \boldsymbol{P}\left(\hat{k}, \boldsymbol{r}_{M}\right) \cdot(\overline{\boldsymbol{I}}-\hat{k} \hat{k}) \\
& \cdot \tilde{\boldsymbol{J}}(\hat{k}) d \hat{k}^{2}
\end{aligned}
$$

of the field probe measuring the radiated AUT field distribution is obtained by translating the plane waves to the field probe position $\boldsymbol{r}_{M}$, where they are superimposed in a weighted manner. $\omega$ is the angular frequency, $T_{L}\left(\hat{k}, \hat{r}_{M}\right)$ is the diagonal FMM translation operator (Coifman et al., 1993;
Chew et al., 2001), and

$$
\boldsymbol{P}\left(\hat{k}, \boldsymbol{r}_{M}\right)=\left(\begin{array}{c}
v_{\mathrm{Pol} 1} \mathrm{FF}_{\mathrm{Pol} 1} \\
v_{\mathrm{Pol} 2} \mathrm{FF}_{\mathrm{Pol} 2}
\end{array}\right)
$$

is the probe correction coefficient, which is used to weight the incident plane waves at the field probe prior to superposition. Due to the far-field characteristic of the plane waves used as equivalent sources, the probe correction coefficient is simply the far-field characteristic of the probe FF multiplied with the antenna factor $v$ converting the measured electric field to the probe output voltage.

In the approach presented so far translations are carried out to every measurement point explicitly, which becomes numerically intensive for large antennas and a high number of measurement points. A multilevel version of the algorithm (Schmidt and Eibert, 2008), related to the multilevel fast multipole method (MLFMM) (Chew et al., 2001), organises the measurement scenario in a multilevel box structure, where translations are carried out to the box centres on the highest level $N$ only, resulting in the spectrum

$$
\tilde{\boldsymbol{J}}_{N}^{i_{N}}(\hat{k})=T_{L}\left(\hat{k}, \hat{r}_{\text {box }}\right)(\overline{\boldsymbol{I}}-\hat{k} \hat{k}) \cdot \tilde{\boldsymbol{J}}(\hat{k})
$$

at the $i_{N}$-th box. The plane waves

$$
\tilde{\boldsymbol{J}}_{n}^{i_{n}}(\hat{k})=\overline{\boldsymbol{D}}_{n}^{i_{n}}\left(\boldsymbol{k}, \boldsymbol{r}_{n}^{i_{n}}\right) \cdot(\overline{\boldsymbol{I}}-\hat{k} \hat{k}) \cdot \tilde{\boldsymbol{J}}_{n+1}^{i_{n+1}}(\hat{k})
$$

are processed through the different levels towards the measurement points recursively using a disaggregation and anterpolation procedure as illustrated in Fig. 1.

Disaggregation is a simple phase shift from the box centre on the higher level to the box centre on the lower level and finally the measurement points. Anterpolation is the adjoint operation to interpolation and reduces the sampling rate (Schmidt and Eibert, 2008; Chew et al., 2001). This is possible since the spectral content of the plane wave spectrum is proportional to the box size on a given level. This reduces the complexity of the algorithm from $\mathcal{O}\left(N^{2}\right)$ to $\mathcal{O}(N \log N)$, $N$ being the number of measurement points. The phase shift and the anterpolation dyad $\overline{\boldsymbol{V}}_{n}(\hat{k})$ are combined in

$\overline{\boldsymbol{D}}_{n}^{i_{n}}\left(\boldsymbol{k}, \boldsymbol{r}_{n}^{i_{n}}\right)=\overline{\boldsymbol{V}}_{n}(\hat{k}) \mathrm{e}^{-\mathrm{j} \boldsymbol{r}_{n}^{i_{n}} \cdot \boldsymbol{k}}$

Arrived on the lowest level, the final phase shift from the box centre on the lowest level to the measurement point is performed and also the probe correction is carried out for a minimum number of plane wave samples, resulting in the probe output voltage

$$
\begin{aligned}
\boldsymbol{U}\left(\boldsymbol{r}_{M}\right)= & -\mathrm{j} \frac{\omega \mu}{4 \pi} \sum_{\hat{k}} W(\hat{k}) \mathrm{e}^{-\mathrm{j} \tilde{\boldsymbol{r}}_{M}^{i_{n}} \cdot \boldsymbol{k}} \boldsymbol{P}\left(\hat{k}, \boldsymbol{r}_{M}\right) \\
& \cdot(\overline{\boldsymbol{I}}-\hat{k} \hat{k}) \cdot \tilde{\boldsymbol{J}}_{0}^{i_{0}}(\hat{k}) .
\end{aligned}
$$

The integrals are evaluated numerically using GaussLegendre quadrature (Abramowitz and Stegun, 1972) with the weights $W(\hat{k})$. 


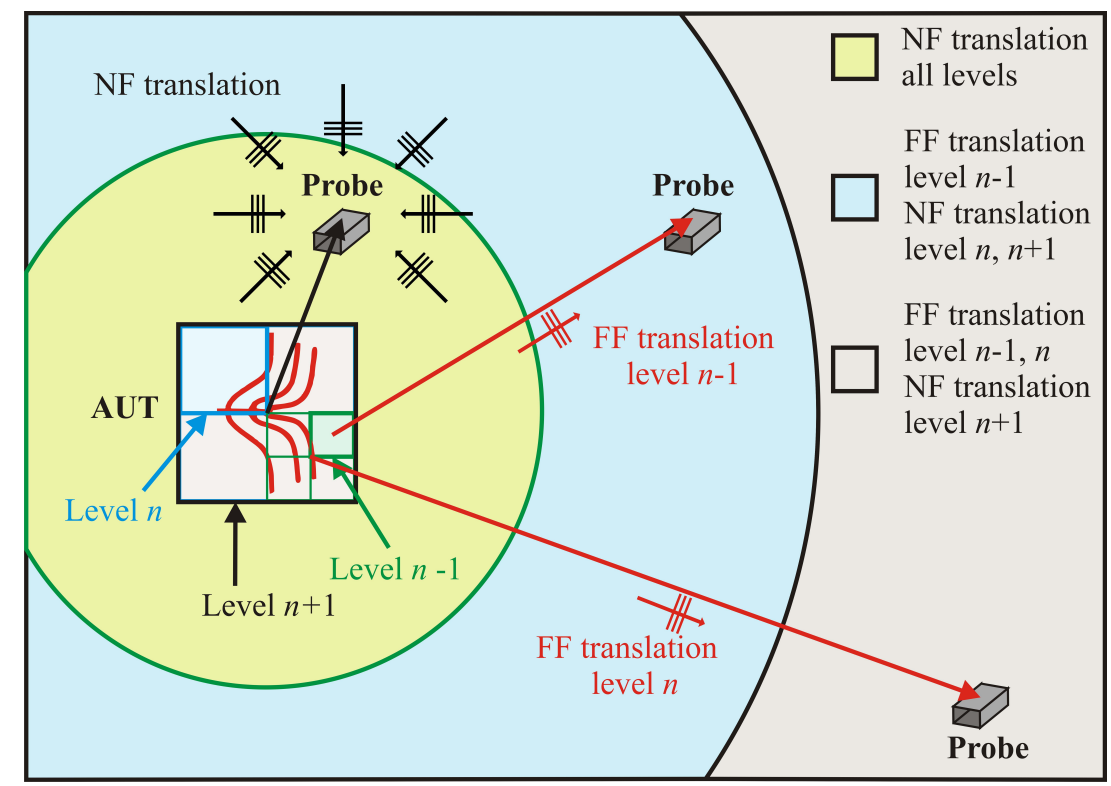

Fig. 2. Near-field and far-field translation ranges.

Electrically large antennas require a huge number of plane wave samples and so iterative solver techniques like the generalised minimum residual method (GMRES) (Saad, 2003) are employed to solve the normal equation on the fly. On the fly means, the probe output voltages are computed from a given set of plane waves for the forward operator without forming a coupling matrix explicitly. The adjoint operator is applied to the result of the forward operator in order to solve the normal system of equations.

\section{Hybrid approach}

For the derivation of the near-field transformation algorithm presented in the previous section, a multipole expansion and a subsequent plane wave expansion have been applied. In order for the plane wave representation to converge, the minimum spheres enclosing AUT and probe must not overlap. For any larger distance the diagonal FMM translation operators can be applied to translate the plane waves to the field probe position. These operators work with a complete set of plane waves and the translations are referred to as near-field translations. For measurement points with a distance larger than the far-field distance

$r_{\text {far }}=\frac{\pi(0.87 d)}{\lambda}$

from the AUT, it is possible to use efficient far-field translations (Chew et al., 2002), which require the translation of a single plane wave in the direction towards the measurement point only. $d$ is the side length of the minimum box enclosing the AUT. Nevertheless the far-field condition is normally

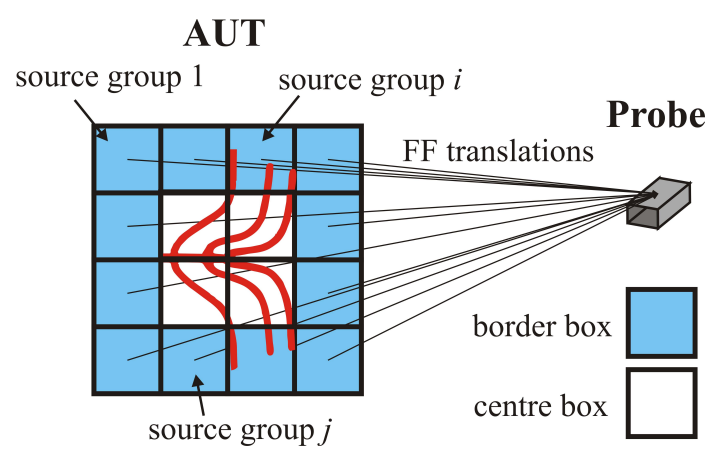

Fig. 3. Source box grouping and far-field translations.

not fulfilled for a near-field measurement and only for some measurement points at the border of a planar scan surface for example, far-field translations might be applicable. In order to facilitate far-field translations, it is possible to recursively subdivide the AUT in smaller source boxes, each of them fulfilling Eq. (7). The different translation ranges for near- and far-field translations resulting thereby are shown in Fig. 2.

Each source box contributes with a plane wave to the total probe voltage at a measurement point $\boldsymbol{r}_{M}$ resulting in a partial voltage

$$
\begin{aligned}
\boldsymbol{U}_{\mathrm{sb}}\left(\boldsymbol{r}_{M}\right)= & -\mathrm{j} \frac{\omega \mu}{4 \pi} \frac{\mathrm{e}^{-\mathrm{j} k\left(r_{M}-r_{\mathrm{sb}}\right)}}{r_{M}-r_{\mathrm{sb}}} \boldsymbol{P}\left(\hat{k}, \boldsymbol{r}_{M}\right) \cdot\left(\overline{\boldsymbol{I}}-\hat{k}_{0} \hat{k}_{0}\right) \\
& \cdot \tilde{\boldsymbol{J}}\left(\hat{k}_{0}\right),
\end{aligned}
$$

with $\boldsymbol{r}_{\mathrm{sb}}$ the source box centre. Therefore a separate set of plane waves is assumed in every border box (see Fig. 3) 


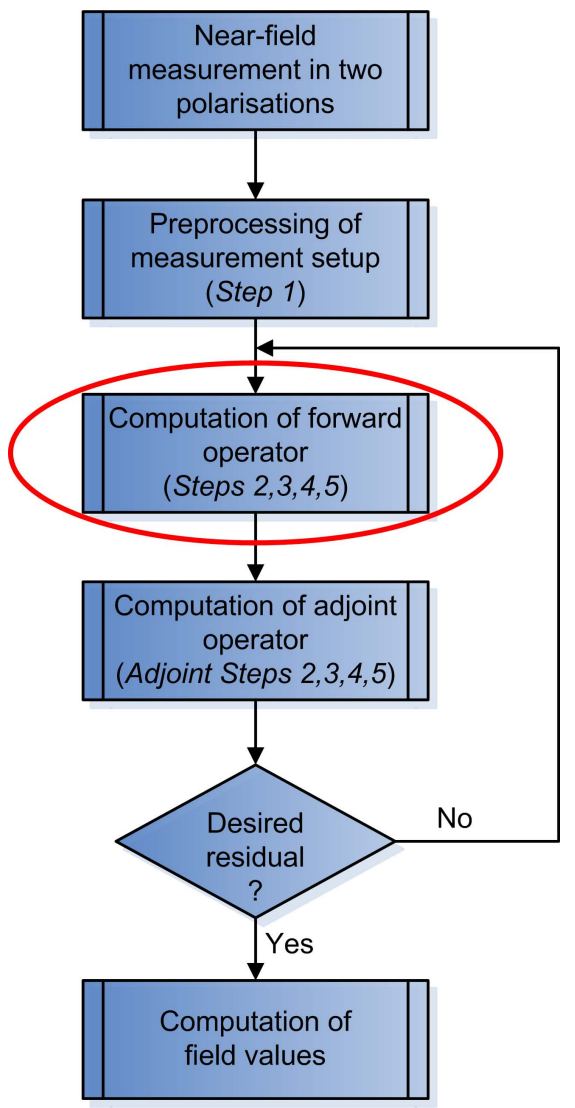

Fig. 4. Flow chart near-field transformation.

as equivalent sources and the plane wave in the direction $\hat{k}_{0}=\left(\boldsymbol{r}_{\boldsymbol{M}}-\boldsymbol{r}_{\mathrm{sb}}\right) /\left|\boldsymbol{r}_{\boldsymbol{M}}-\boldsymbol{r}_{\mathrm{sb}}\right|$ is interpolated. Since it is sufficient to have equivalent sources enclosing the AUT it is not required to have additional sources in the centre boxes. The total probe output voltage

$\boldsymbol{U}\left(r_{M}\right)=\sum_{i} \boldsymbol{U}_{\mathrm{sb}, i}\left(\boldsymbol{r}_{M}, \hat{k}_{0, i}\right)$

is obtained as superposition of all source box contributions.

A typical near-field measurement with subsequent nearfield transformation is described in the flow chart in Fig. 4. First the near-field measurement is carried out for two (usually orthogonal) field polarisations. In the second step the measurement setup is pre-processed, e.g. the multilevel box structure is established and translation and disaggregation operators are pre-computed for an efficient computation of the forward operator. The desired amplitudes of the equivalent sources are determined by the iterative GMRES solver. The matrix-vector-products to determine the base of the Krylov subspace are carried out on the fly by applying the adjoint operator to the result of the forward operator. After each iteration the stop criterion is checked and if the desired residuum is reached field values can be computed from the

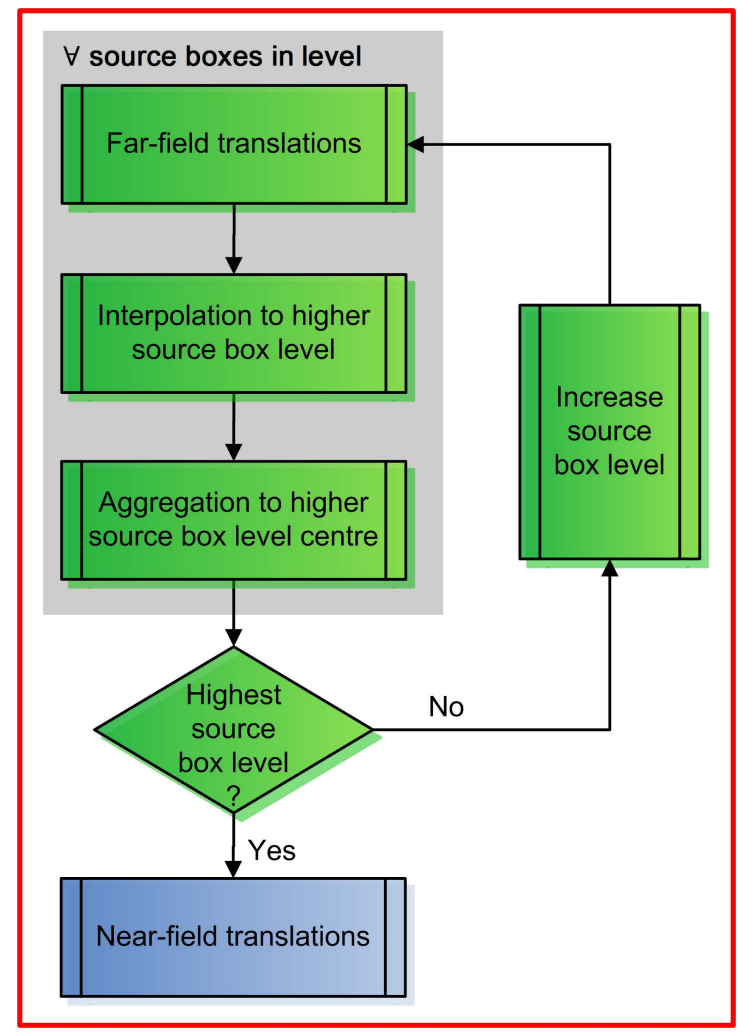

Fig. 5. Flow chart hybrid forward operator.

known equivalent sources. Otherwise further iterations are performed.

To emphasise the novel hybrid approach, the forward operator is described in the flow chart in Fig. 5 in detail. The green boxes show steps of the hybrid approach while blue boxes belong to the standard approach. The formulation starts at the lowest level with the highest number of source boxes. Far-field translations to the measurement points fulfilling the far-field criterion for the source boxes on the given level are performed first. For every measurement point the highest level for which far-field translations may be used is determined in the pre-processing step and translations are preferably carried out on the highest possible level (Tzoulis and Eibert, 2005), since the overall number of translations is smaller for a lower number of source boxes. In the next step the plane wave spectra of the boxes on the given level are interpolated to the sampling rate on the next higher level and aggregated to the centre of the parent box. This is done recursively until arrived on the highest level, where only one box remains enclosing the complete AUT. The plane wave samples of this spectrum are used as equivalent sources in the standard approach and they are used to carry out the nearfield translations in the conventional way, for measurement points not fulfilling the far-field condition with the given number of source box levels. For an increasing number of 


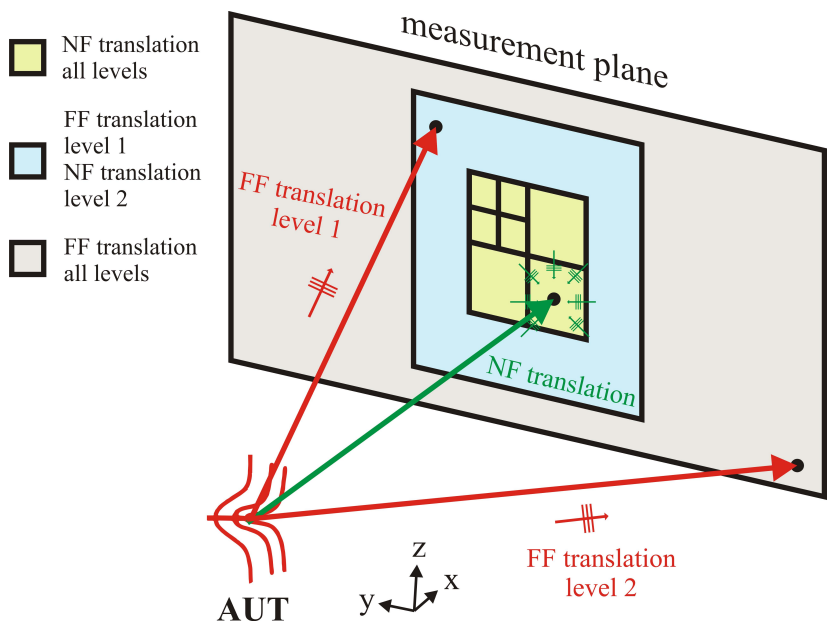

Fig. 6. Planar setup for performance evaluation.

Table 1. Performance of different translation types.

\begin{tabular}{lrrr}
\hline Translation type & Time & \# NF points & \# FF points \\
\hline NF & $19.5 \mathrm{~s}$ & 22801 & 0 \\
NF/FF (1 level) & $8.0 \mathrm{~s}$ & 7377 & 15424 \\
NF/FF (2 levels) & $6.0 \mathrm{~s}$ & 1597 & $15424 / 5780$ \\
FF & $2.0 \mathrm{~s}$ & 0 & 22801 \\
\hline
\end{tabular}

source boxes, the computational effort for the far-field translations is increasing and therefore the number of source box levels has to be chosen carefully in order to find an optimal combination of near- and far-field translations.

The adjoint operator is determined in a similar way to the forward operator utilising aggregation and interpolation as adjoint operations to disaggregation and anterpolation.

\section{Results and performance evaluation}

In this section the functionality and the performance of the presented hybrid near-field transformation algorithm should be shown. Therefore a simple near-field measurement scenario is considered, which was obtained by the electromagnetic field simulator FEKO (http://www.feko.info). Considered is a two-element Yagi-Uda antenna operating at $1.8 \mathrm{GHz}$. A large planar scan surface of $12 \mathrm{~m}$ times $12 \mathrm{~m}$ was chosen allowing to investigate the different translation types in a comfortable way. The principal measurement setup with different translation ranges is shown in Fig. 6.

In order to compare the performance of the different translation types, the measurement plane is chosen such that it is in far-field distance to the AUT. Two transformations, one utilising near-field (NF), the other utilising far-field (FF) translations only, have been carried out to get the minimum and maximum transformation times. The results are shown
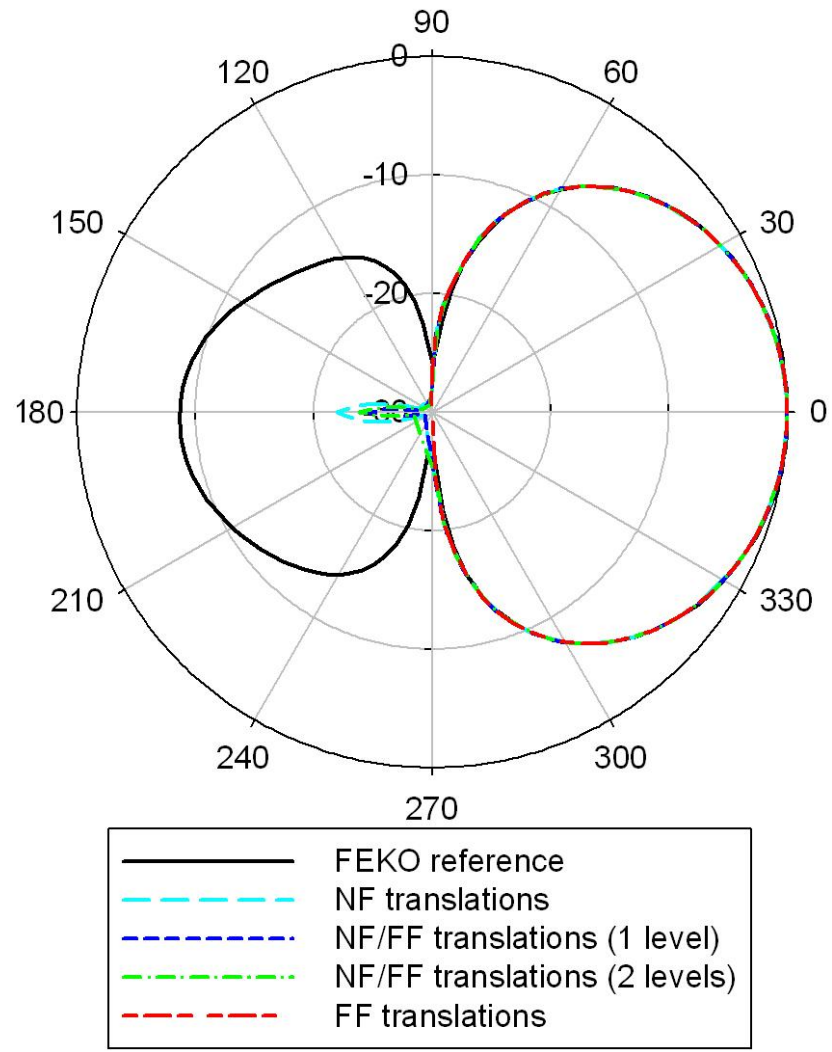

Fig. 7. Comparison of far-field patterns for different translation types (horizontal cut).

in Table 1 and the time in the second column is given for a single solver iteration. For the first hybrid transformation (1 level), NF translations have been used for the centre points of the measurement plane and FF translations for the points at the borders. Around two thirds of the measurement points are treated with FF translations and the computation time could be reduced significantly.

By comparing the times for NF translations only and FF translations only with the hybrid approach it becomes obvious, that the main part of the computation time is spent for $\mathrm{NF}$ translations. For the second hybrid transformation (2 levels), a second source box level is introduced further decreasing the number of NF translations. The transformation time is further decreasing, but it can be seen from the decrease of $2 \mathrm{~s}$ compared to the first hybrid transformation, that FF translations with an increasing number of source boxes also increase the transformation time. In Figs. 7 and 8 the results of the different transformations are shown together with the FEKO reference far-field pattern in a horizontal and vertical cut. Due to the planar measurement surface, the far-field pattern is of course only valid in the forward hemisphere or slightly beyond. The results are all comparable to the reference solution showing no degradation of the transformation accuracy by the hybrid approach. 


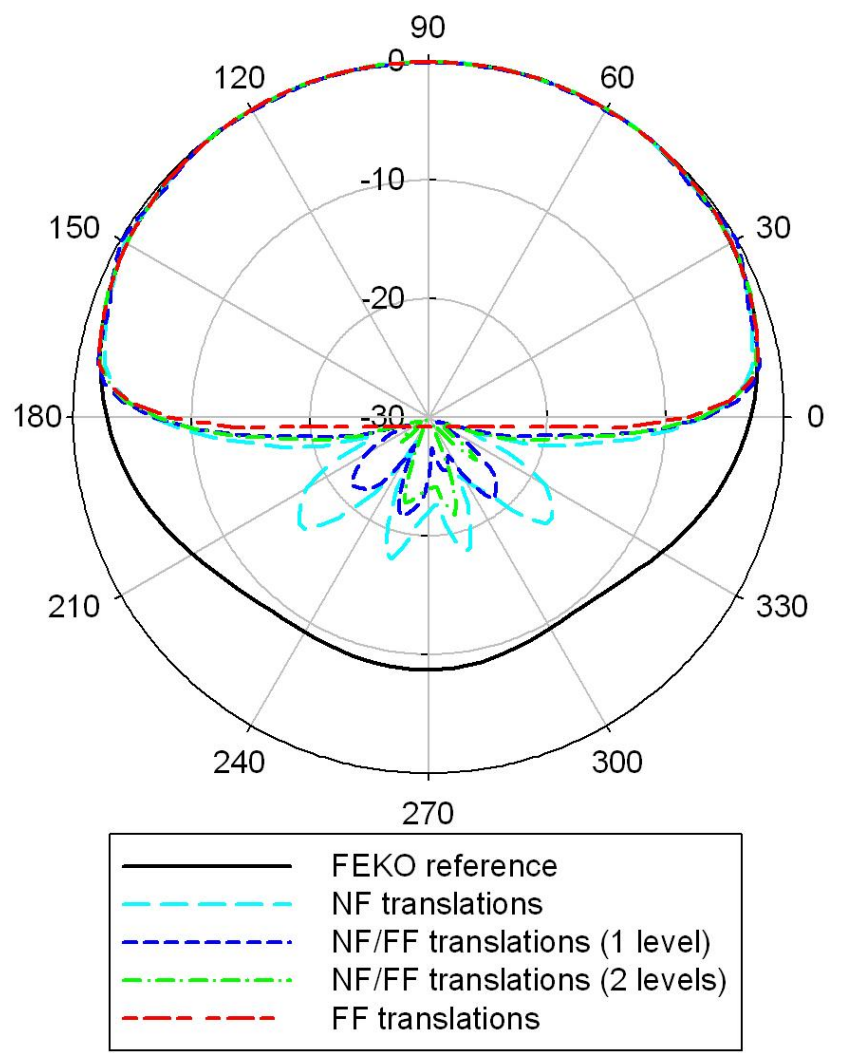

Fig. 8. Comparison of far-field patterns for different translation types (vertical cut).

\section{Conclusions}

A hybrid approach for the near-field far-field transformation employing plane wave expansion and diagonal translation operators has been presented. The algorithm works with a multilevel plane wave representation resulting in an overall complexity of $\mathcal{O}(N \log N), N$ being the number of measurement points. Utilising very efficient far-field translations in combination with the standard near-field translations, the efficiency is further enhanced. With a source box grouping scheme, the required far-field condition can be achieved also for larger antennas. The algorithm has a full probe correction included and is suited for electrically large antennas due to the low complexity. No special requirements for the measurement grid do apply and also irregular sample point distributions can be handled.

\section{References}

Abramowitz, M. and Stegun, I. E.: Handbook of Mathematical Functions, Dover Publications, Inc., New York, 9th edn., 1972.

Chew, W. C., Jin, J. M., Michielssen, E., and Song, J. M.: Fast and Efficient Algorithms in Computational Electromagnetics, Artech House, Inc., 2001.

Chew, W. C., Cui, T. J., and Song, J. M.: A FAFFA-MLFMA Algorithm for Electromagnetic Scattering, IEEE Trans. Antennas Propag., 50, 1641-1649, 2002.

Coifman, R., Rokhlin, V., and Wandzura, S.: The Fast Multipole Method for the Wave Equation: A Pedestrian Prescription, IEEE Antennas and Propag. Mag., 35, 7-12, 1993.

FEKO Suite 5.4, http://www.feko.info, access: 24 February 2009.

Hansen, J. E.: Spherical Near-Field Antenna Measurements, IEE Electromagnetic Wave Series 26, Exeter, UK, 1988.

Kerns, D. M.: Plane-Wave Scattering-Matrix Theory of Antennas and Antenna-Antenna Interactions, National Bureau of Standards, Boulder CO, 1981.

Saad, Y.: Iterative Methods for Sparse Linear Systems, Society for Industrial and Applied Mathematics, 2nd edn., 2003.

Sarkar, T. K. and Taaghol, A.: Near-Field to Near/Far-Field Transformation for Arbitrary Near-Field Geometry Utilizing an Equivalent Current and MoM, IEEE Trans. Antennas Propag., 47, 566$573,1999$.

Schmidt, C. H. and Eibert, T. F.: Multilevel Fast Near-Field FarField Transformation for Electrically Large Antennas, IEEE APS International Symposium, San Diego, USA, 2008.

Schmidt, C. H., Leibfritz, M. M., and Eibert, T. F.: Fully ProbeCorrected Near-Field Far-Field Transformation Employing Plane Wave Expansion and Diagonal Translation Operators, IEEE Trans. Antennas Propag., 56, 737-746, 2008.

Tzoulis, A. and Eibert, T. F.: Efficient Electromagnetic Near-Field Computation by the Multilevel Fast Multipole Method Employing Mixed Near-Field/Far-Field Translations, IEEE Antennas Wireless Propag. Lett., 4, 449-452, 2005.

Yaghjian, A. D.: An Overview of Near-Field Antenna Measurements, IEEE Trans. Antennas Propag., 34, 30-45, 1986. 